\title{
Impatto della lobectomia sulla sopravvivenza di pazienti affetti da carcinoma papillare della tiroide classificati T1b-T2
}

\author{
Maria Grazia Castagna ${ }^{1}$
}

Accettato: 22 luglio 2021 / Pubblicato online: 14 settembre 2021

(c) The Author(s) 2021

\section{Commento a:}

Thyroid lobectomy for T1b-T2 papillary thyroid cancer with high-risk features.

P. Suman, S.N. Razdan, C.E. Wang, M. Tulchinsky, L. Ahmed, R.A. Prinz, D.J. Winchester.

J Am Coll Surg (2020) 230(1):136-144

Il trattamento iniziale del carcinoma papillare della tiroide si basa sulla chirurgia seguita, in casi selezionati, dalla terapia radiometabolica con 131I. In accordo con le linee guida dell'American Thyroid Association [1], il trattamento chirurgico si può avvalere della sola lobectomia in caso di tumori di dimensioni $\leq 4 \mathrm{~cm}$ e in assenza di caratteristiche cliniche e istopatologiche di alto rischio (le metastasi linfonodali e/o a distanza, estensione extratiroidea, invasione vascolare e varianti istologiche aggressive).

Lo studio di Suman e collaboratori [2] ha avuto come obiettivo quello di verificare, in una larga casistica di pazienti con carcinoma papillare della tiroide T1b-T2 sottoposti a lobectomia, la prevalenza di carcinoma papillare T1bT2 con caratteristiche cliniche e istopatologiche di alto rischio e l'impatto di una chirurgia di tipo conservativo sulla sopravvivenza a 5 e 10 anni. Nel gruppo di controllo sono stati inclusi pazienti con carcinoma papillare T1b-T2 senza caratteristiche cliniche e istopatologiche di alto rischio e sottoposti anch'essi a lobectomia.

Sono stati valutati retrospettivamente 8.083 pazienti affetti carcinoma papillare della tiroide suddivisi in due gruppi, il gruppo di studio che includeva 1.552 pazienti in cui la

M.G. Castagna

mariagrazia.castagna@unisi.it

1 Dipartimento di Scienze Mediche, Chirurgiche e Neuroscienze, Università di Siena, Siena, Italia lobectomia veniva considerata inadeguata, vista la presenza di fattori di rischio aggiuntivi, e 6.531 pazienti in cui veniva considerata adeguata, vista l'assenza di caratteristiche cliniche e istopatologiche di alto rischio [2].

Dal confronto tra i due gruppi (lobectomia adeguata, LA, e non adeguata, LNA) emergeva come la sopravvivenza a 5 e 10 anni era significativamente inferiore nel gruppo di pazienti trattati con LNA $(94,7$ vs $97,5 \%$ a 5 anni e 88,5 vs $92,4 \%$ a 10 anni).

Fattori predittivi di una minore sopravvivenza erano: 1) lobectomia non adeguata; 2) età $\geq 45$ anni; 3) sesso maschile; 4) comorbidità; 5) assicurazione governativa; e 6) basso reddito.

Un ulteriore confronto è stato effettuato con un gruppo di pazienti affetti da carcinoma papillare della tiroide con caratteristiche cliniche e istopatologiche di alto rischio e trattati con tiroidectomia totale e terapia radiometabolica con 131I. Anche in questo caso la sopravvivenza dei pazienti trattati con LNA era significativamente inferiore rispetto al gruppo di pazienti trattati con tiroidectomia totale e radioiodio $(94,7$ vs $97,1 \%$ a 5 anni e 88,5 vs $92,3 \%$ a 10 anni.

In conclusione, i dati di questo studio confermano l'inadeguatezza di una chirurgia conservativa nei tumori con caratteristiche cliniche e istopatologiche di alto rischio, indipendentemente dalle dimensioni del tumore primitivo. Evidenziano, inoltre, come la lobectomia può non essere il trattamento ottimale in un sottogruppo di pazienti con tumore di dimensioni $\leq 4 \mathrm{~cm}$ che, nel presente studio rappresentavano circa il 19\% dell'intera casistica. Ciò suggerisce la necessità di un'attenta valutazione pre-chirurgica al fine di identificate correttamente il sottogruppo di pazienti per i quali, vista la presenza di caratteristiche cliniche e istopatologiche di alto rischio, la lobectomia può rappresentare un trattamento non adeguato. Bisogna comunque sottolineare come non 
sempre questi pazienti vengano correttamente identificati alla valutazione pre-chirurgica. Un recente lavoro condotto su pazienti elegibili dopo attenta valutazione pre-chirurgica per la lobectomia ha dimostrato come, in circa il 50\% di questi pazienti, alla valutazione post-chirurgica veniva rilevata la presenza di almeno una caratteristica di alto rischio [3]. In tal caso, una volta correttamente definita l'estensione della malattia, può essere necessario effettuare la lobectomia controlaterale seguita dalla terapia radiometabolica con 131I, ove indicata.

Funding Note Open access funding provided by Università degli Studi di Siena within the CRUI-CARE Agreement.

Nota della casa editrice Springer Nature rimane neutrale in riguardo alle rivendicazioni giurisdizionali nelle mappe pubblicate e nelle affiliazioni istituzionali.

Open Access This article is licensed under a Creative Commons Attribution 4.0 International License, which permits use, sharing, adaptation, distribution and reproduction in any medium or format, as long as you give appropriate credit to the original author(s) and the source, provide a link to the Creative Commons licence, and indicate if changes were made. The images or other third party material in this article are included in the article's Creative Commons licence, unless indicated otherwise in a credit line to the material. If material is not included in the article's Creative Commons licence and your intended use is not permitted by statutory regulation or exceeds the permitted use, you will need to obtain permission directly from the copyright holder. To view a copy of this licence, visit http://creativecommons.org/licenses/by/4.0/.

\section{Bibliografia}

1. Haugen BR, Alexander EK, Bible KC et al (2016) 2015 American Thyroid Association management guidelines for adult patients with thyroid nodules and differentiated thyroid cancer: the American Thyroid Association Guidelines Task Force on Thyroid Nodules and Differentiated Thyroid Cancer. Thyroid 26(1):1-133

2. Suman P, Razdan SN, Wang CE et al (2020) Thyroid lobectomy for T1b-T2 papillary thyroid cancer with high-risk features. J Am Coll Surg 230(1):136-144

3. Bakkar S, Al-Omar K, Donatini G et al (2021) Postoperatively determined high-risk histopathologic features in papillary thyroid carcinoma initially eligible for thyroid lobectomy: a game changer. Endocrine. https://doi.org/10.1007/s12020-021-02788-w 